\title{
Balkanologie
}

Balkanologie Revue d'études pluridisciplinaires

Vol. VII, $n^{\circ} 1$ | 2003

Volume VII Numéro 1

\section{La crise macédonienne (1991-1995) et la question des slavophones en Grèce}

\section{Athéna Skoulariki}

\section{(2) OpenEdition}

12 Journals

Édition électronique

URL : http://journals.openedition.org/balkanologie/479

DOI : $10.4000 /$ balkanologie.479

ISSN : 1965-0582

Éditeur

Association française d'études sur les Balkans (Afebalk)

Édition imprimée

Date de publication : 1 juin 2003

Pagination : 137-146

ISSN : 1279-7952

\section{Référence électronique}

Athéna Skoulariki, «La crise macédonienne (1991-1995) et la question des slavophones en Grèce »,

Balkanologie [En ligne], Vol. VII, nº 1 | 2003, mis en ligne le 18 février 2009, consulté le 17 décembre 2020. URL : http://journals.openedition.org/balkanologie/479 ; DOI : https://doi.org/10.4000/

balkanologie. 479

(c) Tous droits réservés 


\title{
LA CRISE MACÉDONIENNE [1991-1995] ET LA QUESTION DES SLAVOPHONES EN GRÈCE ${ }^{1}$
}

\author{
Athéna Skoulariki*
}

Dans les années 1990, la vieille question macédonienne a déclenché une crise diplomatique avec des résultats consternants tant pour la situation économique et la cohésion sociale en République de Macédoine que pour la qualité de la démocratie en Grèce.

$\mathrm{Au}$ cœur du litige résidait une question de dénomination. L'État grec refusa formellement la reconnaissance internationale de l'Ancienne République Yougoslave de Macédoine sous son appellation constitutionnelle. La diplomatie grecque redoutait la monopolisation par le jeune État limitrophe du nom et du patrimoine historiques macédoniens. Par une mobilisation impressionnante, le public grec revendiqua même l'association exclusive de la Macédoine et de son histoire millénaire à la Grèce.

La presse française parlait alors d'un conflit politico-sémantique. Or les objections grecques n'étaient pas d'ordre symbolique, comme l'insistance sur l'antiquité pouvait le laisser entendre. Derrière la rhétorique sur l'usurpation du patrimoine hellénique couvaient des inquiètudes envers l'irrédentisme macédonien et la revendication d'une minorité nationale en Grèce du nord.

L'objet de cet article sera d'examiner les deux dimensions du problème : d'une part, l'importance de la question minoritaire dans le contentieux diplomatique entre la Grèce et la République de Macédoine au début des années 1990, et, d'autre part, la réaction des slavophones grecs déchirés entre deux discours nationaux mutuellement exclusifs. 


\section{LES SLAVOPHONES EN GRÈCE}

Les noms sont porteurs de sens. Leur poids historique peut fonder chez les peuples un sentiment d'appartenance ou d'exclusion. La controverse au sujet du nom de la Macédoine est d'autant plus grave qu'elle touche, outre la République ex-yougoslave, la dénomination de la population slavophone en Macédoine grecque. Enjeu crucial entre les nationalismes concurrents, l'appellation de cette population varie selon le contexte historique et la source de l'énonciation.

Les slavophones en Grèce se définissent le plus souvent comme dopioi Makedones [Macédoniens indigènes, autochtones] en grec, et Makedonci dans leur propre langue. Jusqu'à la Seconde Guerre mondiale, un grand nombre parmi eux se définissait comme "Bulgares"2.

Dans le discours officiel contemporain les termes employés sont "Grecs bilingues" ou "Grecs slavophones". La gauche semble préférer le terme "Slavomacédoniens". Cette appellation était d'ailleurs employée par la diplomatie grecque dans l'entre-deux-guerres et dans les années 1980. On désignait également les citoyens de la République Socialiste de Macédoine comme "Slavomacédoniens", jusqu'à l'invention du nom vulgaire "Skopjens". À la fin des années 1990, les activistes du mouvement minoritaire ont introduit le néologisme "Macédoniens ethniques" [ethnikoi Makedones] en traduisant le terme anglais ethnic, qui pourtant en grec signifie tout simplement "national".

Aucun emploi de nom n'est neutre ; toutes les appellations ont des connotations politiques et idéologiques. Nous avons opté dans cette recherche pour le substantif "slavophones" afin de décrire une réalité socio-linguistique sans porter préjudice à l'identité nationale des individus qui la composent. Notre choix est opérationnel et certainement pas normatif.

Il n'existe pas de statistique fiable sur le nombre des slavophones Macédoniens en Grèce. Les 81984 citoyens recensés en 1928 et les 41017 recensés en 1951 sont les seuls chiffres officiels que nous possédons, mais ils étaient très inférieurs à la réalité 3 . De nos jours, il s'agit de personnes pratiquement bi-

\footnotetext{
2 Pour plus d'informations sur les dénominations et les identités, voir Karakasidou (Anastasia), "Politicizing Culture : Negating Ethnic Identity in Greek Macedonia", Journal of Modern Greek Studies, 11,

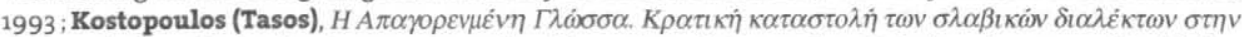
$\varepsilon \lambda \lambda \eta \vee к \dot{\eta}$ M $\alpha \kappa \varepsilon \delta o v i \alpha$ [La langue proscrite. Répression étatique des dialectes slaves en Macédoine grecque], Athènes : Mavri Lista, 2000, pp. 33-45.

${ }_{3}$ D'après les estimations de I. Michailidis, le nombre de slavophones au milieu de la décennie 1920 serait de 160 ooo personnes, dont 101 101, selon une statistique confidentielle, habitaient les départements de



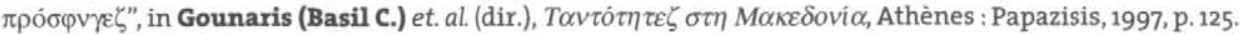
Pour un exposé comparatif des résultats des recensements officiels, ainsi que des statistiques confidentielles de l'administration publique et des Services spéciaux, voir Kostopoulos (Tasos), op. cit., pp. 23-33, 222-224.
} 
lingues, majoritairement concentrées dans les départements de Florina, Kastoria et Pella en Macédoine grecque occidentale. Des villages de slavophones chrétiens subsistent aussi dans les contrées montagneuses de Serres, de Drama et de Kilkis.

Les slavophones ne jouissent pas d'un statut de minorité reconnue et leur langue, un dialecte slavo-macédonien qu'on appelle communément dopi(k)a (local, indigène), n'est pas enseignée 4 . Le nationalisme grec insiste sur le fait que ce n'est pas la langue, mais le sentiment national qui importe et que les slavophones macédoniens ont, à plusieurs reprises, prouvé leur attachement à la Grèce. Dans la pratique, cependant, un effort conscient et organisé a été entrepris dès 1913 de la part de l'État pour gommer le particularisme local. La population slavophone a été longtemps considérée "suspecte" du point de vue de sa loyauté nationale et subissait de manière systématique la méfiance des autorités.

\section{LES AMBIGUITTÉS HISTORIQUES}

La raison de la circonspection officielle envers les slavophones n'est autre que l'incertitude quant à l'intégration nationale des régions de la Macédoine incorporées à l'État grec ${ }^{5}$. Au cours du XX ${ }^{\text {ème }}$ siècle, les États balkaniques ont cherché à homogénéiser leurs territoires. Les ravages des guerres successives, l'échange de populations entre la Bulgarie et la Grèce en 1919 et l'installation en Macédoine grecque des réfugiés grec-orthodoxes de l'Asie Mineure après 1923 ont radicalement modifié la constitution ethnique de la région.

Pendant la guerre civile qui a suivi la Seconde Guerre mondiale, un grand nombre de slavophones ont rejoint les rangs des communistes grecs. La défaite de l'Armée Démocratique en 1949 a obligé les combattants slavomacédoniens,

4 La langue slave-macédonienne ne fut standardisée et dotée d'une littérature écrite qu'après 1944 en tant que langue nationale de la Macédoine yougoslave. Sur les dialectes de la Macédoine grecque voir

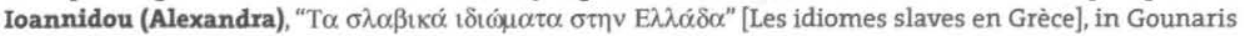
(Basil C.) et. al. (dir.), op. cit., p.89-101.

5 Les territoires en question représentent la pomme de discorde des nationalismes balkaniques au cours des deux derniers siècles. Les trois vilayets de l'époque ottomane étaient habitès par une diversité de peuples qui se sont tardivement engagés dans les mouvements nationaux de la fin du XIX ${ }^{\text {ème }}$ siècle. Parmi eux, les slavophones chrétiens qui habitaient majoritairement la zone médiane et le nord de la Macédoine sont devenus la cible de la concurrence gréco-bulgare. Leur alignement avec l'un ou l'autre camp relevait plus des rapports de force dans chaque localité que de la cristallisation d'une conscience nationale. Les frontières actuelles datent de 1913. À l'issue des deux guerres balkaniques, la Macédoine ottomane a été partagée entre la Grèce ( $51 \%)$, la Serbie (38\%), la Bulgarie (10\%) et l'Albanie (1\%). Voir Lory (Bernard), “Approches de l'identité macédonienne", in Chiclet (Christophe), Lory (Bernard), éds., La République de Macédoine, Paris : L'Harmattan / Les Cahiers de Confluences, 1998 ; Kofos (Evangelos), "National Heritage and National Identity in Nineteenth - and Twentieth-Century Macedonia", European History Quarterly, (19), 1989, pp. 229-267. 
tout comme des milliers d'autres Grecs, à se réfugier dans les pays de l'Est. Une partie s'est installée dans la République Populaire de Macédoine. Enfin, l'émigration économique des années 1950-1960 a également contribué à la diminution de la population slavophone.

Malgré ces bouleversements démographiques, l'État grec ne sentait pas ses Nouvelles terres tout à fait sécurisées. La présence de slavophones, bien que très réduite par rapport au passé, pouvait donner lieu à une contestation de la souveraineté nationale. L'occupation bulgare lors des deux Guerres mondiales, le projet du Komintern pour une Macédoine "unie et indépendante" et les convoitises yougoslaves sur la Macédoine de l'Égée représentaient des antécédents inquiétants. La réponse a été double : au niveau international, l'altérité linguistique et culturelle d'une partie de la population locale a été niée ; à l'intérieur, la politique d'assimilation a repris à partir des années 1950 et jusqu'à la fin des années $1970^{6}$.

Les mesures adoptées visaient aussi bien à imposer aux slavophones de parler la langue grecque, qu'à leur faire oublier la leur. Les autorités locales interdisaient l'usage public de cet idiome et les habitants étaient contrôlés par la police s'ils osaient chanter leurs chants traditionnels. Dans le contexte de la guerre froide, l'association du "danger slave" au "danger communiste" a fait doubler la méfiance des autorités. La surveillance de la population par les services secrets était accablante, dans une région qui souffrait des séquelles de la guerre civile.

Les premiers signes de démocratisation n'ont été perçus par la population slavophone qu'en 1981 avec l'arrivée du parti socialiste au pouvoir?. La langue locale pouvait être parlée publiquement sans conséquences. Cependant, l'État n'a autorisé le retour qu'aux seuls réfugiés politiques "Grecs d'origine". En introduisant un critère ethnique, cette exception discriminatoire reconnaissait indirectement la réalité d'une question minoritaire en Macédoine grecque.

\section{LE CONTENTIEUX X̀ PROPOS DE LA MINORITÉ}

La crise macédonienne au début des années 1990 a coïncidé en Grèce avec une période d'instabilité politique. La polémique au sujet de la Macédoine a

6 Voir Kostopoulos (Tasos), op. cit., pp. 222-283. La répression linguistique était la norme depuis les années 1930. Pendant la dictature du Général Metaxas (1936-1940), des persécutions sévères avaient eu lieu, et les dialectes non grecs avaient été prohibés sous peine d'amende, voire d'incarcération ; Ibid., pp. $162-180$.


Oikonomikos Tahydromos, 04/02/93. 
causé la scission du parti de la droite alors au pouvoir et la division du monde politique en "réalistes" et "intransigeants".

En 1995, deux ans après sa défaite électorale, l'ancien premier ministre $\mathrm{K}$. Mitsotakis a fait un aveu inattendu : la "clé du litige" entre Athènes et Skopje n'était point l'appellation, mais la question de la "non-existence de minorité slavomacédonienne" en Grèce ; il affirma que "l'enjeu était d'éviter la création d'un deuxième problème minoritaire, en Macédoine occidentale" 8 .

Malgré tout, la question minoritaire, peu discutée publiquement pendant la crise, a été savamment laissée dans l'ombre par la plupart des analystes et des politiciens, après même ces aveux. Un passé trop chargé, beaucoup de nondits et des pratiques politiques commandées par la raison d'État pesaient lourd sur ce sujet qu'on préférait esquiver, plutôt que de réveiller de vieux démons.

Depuis la fin des années 1970, les déclarations répétées des responsables de Skopje au sujet de la présumée "minorité macédonienne" irritaient les gouvernements grecs qui voyaient dans cette politique un effort d'inscrire des droits pour des revendications territoriales et d'intervenir dans les affaires intérieures de la Grèce. La diaspora macédonienne aux États-Unis, au Canada et en Australie s'adonnait à une propagande irrédentiste de plus en plus agressive. En 1990, en République de Macédoine, plusieurs partis et organisations ont vu le jour, aspirant au vieux rêve de l'unification du peuple macédonien.

La situation se radicalisait. Durant l'été 1990, des nationalistes macédoniens avaient bloqué à plusieurs reprises la frontière avec la Grèce et distribuaient des cartes de la Grande Macédoine. Les autorités macédoniennes ont alors décidé l'internationalisation de la question minoritaire. La Grèce a été dénoncée auprès de l'ONU et de la CSCE pour la non-reconnaissance "de l'identité nationale de la minorité macédonienne".

La réponse de la Grèce a été vive. Les derniers slavophones de conscience non-grecque étaient censés avoir quitté le pays à la fin de la guerre civile, et depuis "pas un individu ou un groupe d'individus de cette minorité "fantôme" n'a, pendant toutes ces années, déposé la moindre plainte contre l'État grec pour de telles violations [aux droits de l'homme]"9.

Deux citoyens grecs originaires de la Macédoine occidentale ont réussi alors à démentir cet argument diplomatique ; à une conférence de presse organisée au cours de la session de la CSCE à Copenhague, ils se sont déclarés

8 Mitsotakis (Konstantinos), "Preface", in Skylakakis (Th.), $\Sigma \tau o$ óvo $\mu \alpha \tau \eta \zeta$ M $\alpha \kappa \varepsilon \delta o v i \alpha \zeta$, Athènes: Elliniki Euroekdotiki, 1995, p. 3. Bien qu'il considérât la question minoritaire "émoussée" avec le temps et de nos jours "essentiellement inexistante", Mitsotakis admettait que tant que les "tendances irrédentistes persistent" en République voisine, le problème pouvait être de nouveau attisé ; ibid., p. 4 . Le premier problème minoritaire auquel il fait allusion est celui de la minorité musulmane en Thrace occidentale.

9 "Déclaration du chef de la délégation grecque en réponse à l'intervention yougoslave à la session plénière de la CSCE ", Conférence sur la dimension humaine, CSCE, Copenhague, 5-29 juin 1990. 
"membres de la minorité macédonienne en Grèce" et ont réclamé le respect de leurs droits. Depuis, un groupe d'activistes s'est organisé pour revendiquer la reconnaissance de la langue et de la culture macédoniennes. Les autorités grecques minimisaient leur présence et parlaient de "cas isolés"10.

La Grèce avait une approche juridique de la question des minorités : l'État ne reconnaissait des obligations que si elles découlaient d'un traité international. On évitait ainsi d'envisager le vrai problème, à savoir l'existence éventuelle d'une communauté slavophone aspirant à des droits collectifs.

Sur ce sujet l'État grec n'avait pas une position conséquente. Alors qu'en 1924, une minorité bulgare a été reconnue, et qu'en 1926 un manuel scolaire a été préparé pour l'apprentissage de la langue "macédonoslave"11, après la Seconde Guerre mondiale, la diplomatie grecque insistait sur le dogme de la "question inexistante". Au début des années 199o, le Premier ministre admettait tout au plus l'existence de " 20000 Grecs bilingues vivant dans les régions frontalières"12.

La Constitution de la République de Macédoine prévoyait que l'État s'engageait à veiller "au statut et aux droits [des] membres du peuple macédonien dans les pays voisins, (...) [à] assiste[r] leur développement culturel et [à] fai[re] avancer les liens avec eux". Ces dispositions ont été jugées "irrédentistes" par la Grèce, qui a réussi à bloquer la reconnaissance du pays par la Communauté Européenne ${ }^{13}$. Athènes défiait les autorités de Skopje qui déclaraient ne pas avoir de visées territoriales, mais qui insistaient pour la reconnaissance d'une minorité nationale en Grèce.

10 L'amalgame entre les termes de groupe ethnique, de minorité ethnique et de minorité nationale ajoutait à la confusion générale quant aux militants et à leurs objectifs. Les activistes n'employaient pas de façon cohérente les termes "identité ethnique" et "identité nationale". D'autre part, bien que l'existence d'une conscience nationale macédonienne chez un nombre significatif des slavophones soit disputée, la réalité d'un groupe ethnique distinct ayant sa propre langue et culture est difficilement contestable. Ceux qui reconnaissent la réalité de la population slavophone, confondent souvent le terme générique "minorité", qui décrit une différentiation culturelle (ethnique, linguistique, religieuse, etc.), avec la catégorie "minorité nationale", qui exprime une revendication politique. Ainsi Veremis et Dragoumis (Veremis (Thanos M.), Dragoumis (Mark), eds., Historical Dictionary of Greece, London : Scarecrow Press, 1995, pp. 118-119) écrivent sur la Macédoine grecque : "Les quelques familles restées en Macédoine occidentale qui parlent un idiome slavon ont opté de se considérer Grecques; il n'y a donc pas actuellement de minorité slavomacédonienne en Grèce". Pour capter la nuance il faut souvent pouvoir lire entre les lignes. En 1991, à propos du rapport du State Department, le premier ministre K. Mitsotakis avait affirmé : “Cette minorité en tant que minorité nationale n'existe pas, c'est une minorité-fantôme" (Procès-verbaux de l'Assemblée Nationale, séance du 12 février 1991). Quant aux nationalistes engagés, ils refusaient l'existence d'une minorité "macédonienne" se fondant sur l'argument formaliste qu'il n'existe pas, en premier lieu, de nation "macédonienne".

11 Voir Michailidis (Iakovos), "Minority Rights and Educational Problems in Greek Interwar Macedonia : The Case of the Primer Abecedar", Journal of Modern Greek Studies, 114 (2), 1996.

12 Discours du premier ministre K. Mitsotakis à l'Assemblée Nationale, le 12 février 1991.

13 Malgré la révision de la Constitution en janvier 1992, l'article 49 est resté inchangé, à l'exception d'une phrase qui fut ajouté prévoyant que "la République n'interviendra pas dans les droits souverains des autres États, ni dans leurs affaires intérieures". La Grèce a considéré la révision insuffisante. 


\section{LE DISCOURS PUBLIC EN GRÈCE}

L'appropriation du nom Macédoine par plus d'un pays est le vestige d'une époque - pas si éloignée d'ailleurs - où plus d'un peuple vivait sur un seul territoire ; cette réalité refoulée démentit les discours nationalistes réciproques et relativise la légitimité des frontières contemporaines.

Pour abjurer ce rappel ennuyeux, les défenseurs de l'hellénicité de la Macédoine n'ont pas hésité à supprimer l'extrême complexité de l'histoire balkanique par un aphorisme : "Ils parlent de trois Macédoines. Or il n'y a eu et il n'y aura toujours qu'une seule Macédoine, la Macédoine grecque !"14. Réconfortante et schématique, cette interprétation a dominé le discours public pendant la crise des années 1990. La campagne d'information sur la Macédoine cherchait impérativement à minimiser la présence historique des non-grécophones, et à argumenter sur la continuité de la présence nationale grecque dans la région.

En juin 1990 la diplomatie grecque expliquait son silence pendant 45 ans vis-à-vis des "provocations de Skopje" en concluant que : "l'élément étranger slave, en conséquence de son rôle pendant l'Occupation et la Guerre civile, avait abandonné le pays en masse et la perspective de sa manipulation par un pays voisin, afin de menacer la sécurité et l'intégrité [de la Grèce], était éloignée"15.

Ce texte évoque parfaitement le stéréotype de la minorité vue comme agent de déstabilisation au service des convoitises étrangères. Dans tous les pays des Balkans, les minorités nationales sont conceptualisées à travers la métaphore du "cheval de Troie" : un corps tiers introduit au sein de la nation qui se tourne inexorablement contre elle ${ }^{16}$.

L'attitude du monde politique n'était pas uniforme. Les nationalistes - regroupés majoritairement à la droite, mais pas exclusivement - évitaient de reconnaître l'existence de n'importe quelle forme d'altérité et insistaient sur l'unité intrinsèque de la nation grecque.

14 Cf. article de Martis (Nikolaos), To Vima, 22/03/92.

15 "Statement by the Head of the Greek Delegation in Reply of the Yougoslav Intervention at the Plenary Session of June 22, 1990", Conference on Human Dimension, CSCE : Copenhague, 5-29 juin 1990.

16 L'altérité ethnique est redoutée dans la mesure où elle met en question l'homogénéité et donc la cohésion nationale, le fondement même de la légitimation de l'État-nation. C'est, en ce sens, inhérent à cette forme étatique de renier la diversité culturelle (ethnique, linguistique ou religieuse) au sein de la nation. Or, paradoxalement, les minorités nationales sont formées par les tentatives mêmes des États nationaux à assimiler les groupes minoritaires. Car les nationalismes engendrent des contre-nationalismes, de la part des groupes qui se sentent étrangers à la culture dominante et pour cela exclus du corps national. Le cercle est alors bouclé : “Le nationalisme”, note Loring Danforth, "en égalisant la loyauté à l'Etat avec l'appartenance à la nation, transforme par définition les membres des minorités nationales en ennemies de l'Etat". Danforth (L.), The Macedonian Conflict, Princeton University Press, 1995, p. 21. 
La gauche et les libéraux/pro-européens favorisaient au contraire le pluralisme et se portaient en défense des droits des groupes minoritaires. Les réflexes de crispation nationaliste n'étaient toutefois pas rares. Le parti socialiste oscillait entre son idéologie de gauche et son profil "patriotique". Les leaders du mouvement exprimaient leur méfiance par des arguments politiques et en apparence non ethnocentriques: "Dans chaque minorité, d'autres minorités secondaires apparaissent. Ceci se perpétue sans aucune limite. Et finalement on risque la dissolution de la construction européenne", jugeait Andreas Papandreou ${ }^{17}$.

Il est évident, selon ces propos, que les minorités étaient vues à travers le prisme des antagonismes régionaux et que le respect des libertés démocratiques reculait devant des considérations de défense nationale. Aucune mention n'était faite aux difficultés que ces citoyens rencontraient à cause de leur différence ethnolinguistique, ni à leurs aspirations légitimes à sauvegarder leur patrimoine culturel. Enveloppée sous un discours nationaliste affectif ou excusée en invoquant des raisons stratégiques, la méfiance envers les minorités dominait le débat en Grèce.

Le discours de la presse confirme l'absence des slavophones de l'espace public grec. Au tout début de l'affaire, de 1990 à 1991, les altercations entre Athènes et Belgrade n'occupaient pas plus de deux ou trois paragraphes dans les pages intérieures des journaux. En s'alignant aux explications du porte-parole du Ministère des Affaires Etrangères, les journaux grecs réfutaient absolument les thèses yougoslaves comme "inadmissibles et ignorant l'histoire"18.

Il a fallu attendre le mois de février 1991 pour que la question macédonienne inquiète sérieusement les responsables grecs au point d'occuper à gros titres la une des quotidiens. Dans le rapport annuel du State Department des États-Unis sur le respect des droits de l'Homme à travers le monde, l'existence en Grèce d'une "minorité slave-macédonienne" a été mentionnée.

Le rapport a provoqué un tollé. Il a été comparé à une "bombe" ou à des "coups de couteau dans le dos"19. La presse fustigeait la politique américaine et le "Nouvel Ordre Mondial" en s'accordant aux thèses officielles qu'il n'y a ni minorité, ni question macédoniennes. Un "Réseau de propagande de Skopje aux Etats-Unis" serait responsable de ce "document anti-hellène" 20 .

Ce rapport, qui a tant outré la Grèce, ne contenait pourtant rien d'inexact. Mais, à l'époque, la seule mention à une population slavophone, dont l'existence était ignorée par la majorité des Grecs, suffisait pour susciter l'indignation générale.

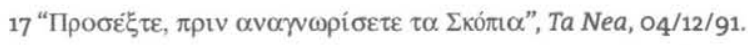

18 To Vima, 24/06/9o. Voir également Ta Nea, 26/06/90; Mesimvrini, o6/o7/9o.

19 Respectivement Eleftherotypia et Eleftheros Typos, 02/02/91.

20 Eleftheros Typos, 02/02/91. 
Cette affaire, bien que vite oubliée, n'a pas été sans conséquences. L'introduction de la question macédonienne par le biais américain, dont les mobiles obscurs étaient toujours exagérés en Grèce, a été déterminante.

Cependant la question de la minorité était un sujet sur lequel l'attention grecque s'est focalisée au début, et qui a été éludé par la suite. Dans le discours officiel le mot minorité n'est presque plus proféré après 1991. On préférait visiblement évoquer la question sous les termes plus allusifs de "propagande hostile" ou d'"irrédentisme". La presse se montrait tout autant allergique à cette notion que l'État. Il n'y avait eu aucun reportage et la question était présentée comme un pur produit de la "propagande de Skopje".

L'absence d'investigation et les rares références à ce sujet extrêmement épineux ne peuvent pas être interprétées comme aléatoires de la part des journalistes. Quand ils étaient obligés de parler de la population slavophone, ils le faisaient avec de grandes précautions : le texte était bref, les termes allusifs, les explications faisaient défaut. Il y a une telle gêne que l'information était, finalement, déformée, volontairement ou non.

La presse a également très peu parlé des militants minoritaires, sauf lorsqu'ils étaient traduits en justice ou subissaient les attaques des groupes de "citoyens indignés", selon une expression courante ${ }^{21}$. La distinction manichéenne entre, d'une part, des citoyens bilingues fiers d'être Grecs et, d'autre part, des "agents de Skopje" qui offensait le sentiment national de la majorité restait la seule interprétation proposée au grand public.

\section{LES SLAVOPHONES PENDANT LA CRISE}

À l'issue de la guerre civile et jusqu'aux années 1990, un silence presque total s'était imposé sur tous les aspects de la question macédonienne. L'État, les autorités locales, les médias et même l'église se sont accordés pour faire oublier l'existence même de la population slavophone en Grèce du nord.

En Macédoine occidentale, un climat de tension était entretenu par la forte présence policière dans les fêtes des villages, l'antagonisme toujours vif entre les slavophones et les réfugiés de l'Asie Mineure, ainsi que par l'activité de divers groupes et associations nationalistes.

Conséquence de cette politique tant officielle qu'officieuse, la peur s'était installée parmi la population slavophone en excluant toute revendication de 
sa spécificité linguistique et culturelle, au moins dans l'espace public ${ }^{22}$. La même peur ainsi que le silence ambiant décourageaient par ailleurs les chercheurs qui auraient pensé à enquêter sur ce sujet ${ }^{23}$. Pendant plusieurs décennies, la communauté des slavophones est donc restée cloisonnée dans le microcosme local, persécutée par les autorités et ignorée par la société grecque dans son ensemble.

Cependant, ces conditions particulières ne doivent en aucun cas créer l'impression que les slavophones en Grèce des années 1980 et 1990 formaient une population à part, compacte et homogène. Les attitudes et les opinions qu'ils exprimaient pendant la crise macédonienne en première lecture varient autant que parmi la population globale.

La différence, il faut la chercher derrière les apparences. Nombreux villages slavophones ont participé massivement entre 1992 et 1994 aux grandes manifestations pour "l'hellénicité de la Macédoine". Leur mobilisation était sans doute sincère, étant donné que la quasi-totalité des slavophones partagent l'identité nationale grecque. Le souci de ne pas laisser planer de doutes sur leur propre "grécité" les poussaient certainement à exagérer leur engagement. Quand la période de tension est passée, nombreux ont osé avouer que ces réactions étaient démesurées.

D'autres, à la même époque, ont préféré s'abstenir. S'identifiant comme Macédoniens dans un pays où ce nom avait dorénavant une acception ambiguë, ils se sont trouvés obligés de choisir leur camp. La polarisation entre le nationalisme grec et le nationalisme macédonien a poussé les slavophones à une réflexion sur leurs origines et leur appartenance. Ils ont gagné une nouvelle conscience de leur spécificité culturelle, dépourvue des complexes du passé, sans forcément défier leur adhérence à la "communauté imaginaire" de la nation grecque.

Certains, parmi les slavophones, ont pourtant choisi une voie plus revendicative. La mise en doute de leur loyauté nationale et la négation de leur culture ont créé un sentiment de frustration qui a fini par cimenter leur identité ethnique.

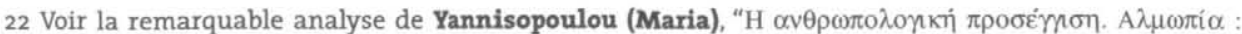
$\pi \alpha \rho \varepsilon \lambda \theta o ́ v, \pi \alpha \rho o ́ v \kappa \alpha \Lambda ~ \mu \varepsilon ́ \lambda \lambda o v "$ [L'approche anthropologique. Almopia : passé, présent et avenir], in EKKE., M $\alpha \kappa \varepsilon \delta$ ovi $\alpha \kappa \alpha \imath$ B $\beta \alpha \lambda \kappa \alpha_{\alpha} v \imath \alpha$, Athènes : Alexandreia, 1998. L'anthropologue explique notamment comment la peur et le silence sont devenus des éléments constitutifs de l'identité des slavophones en vue de leur intégration. Le nom dopioi (autochtones) par lequel ils se distinguent évoque la difficulté d'une affirmation libre de leur identité ethnolinguistique et en même temps une stratégie de protection de leur spécificité : "Le pouvoir central [...] leur a ôté les mots, la parole, "leur langue maternelle"'” (ibid., pp. 359-36o) ; il leur reste la relation à la terre qu'ils habitent, leur origine qui les distingue des réfugiés "intrus".

23 Voir les témoignages des chercheurs linguistes, anthropologues et sociologues au colloque sur les "Dialectes slaves de Macédoine", Athènes, 31 octobre - 1er novembre 1999, actes publiés in KEMO, $\Gamma \lambda \omega \sigma \sigma \iota \kappa \dot{\eta}$ $\varepsilon \tau \varepsilon \rho o ́ \tau \eta \tau \alpha \sigma \tau \eta v E \lambda \lambda \alpha \dot{\alpha} \delta \alpha$ [Altérité linguistique en Grèce], Athènes : Alexandreia, 2001, pp. 141-280.
} 


\section{LE MOUVEMENT MINORITAIRE}

Au début des années 1990, pour la première fois depuis l'intégration de la Macédoine dans l'État grec, des slavophones se sont organisés pour demander des droits minoritaires.

Les politiques d'assimilation et les mesures coercitives ont conduit, à terme, à la politisation d'une partie de la population. "J'ai une conscience nationale macédonienne et une citoyenneté grecque, avec tous les droits et les obligations d'un citoyen grec", déclara, en 1992, l'activiste Christos Sidiropoulos ${ }^{24}$. Alors que certains ont adhéré au nationalisme macédonien, d'autres, beaucoup plus nombreux, aspiraient à la protection de leur langue et de leur culture natales sans remettre en cause leur allégeance à la Grèce.

Mais les médias et les intervenants au débat public ont associé cette évolution à la fameuse "propagande de Skopje". L'existence de citoyens grecs qui revendiquaient une identité nationale macédonienne était inconcevable pour certains et intolérable pour d'autres. Dans les deux cas, le fait était perçu comme une déviance qu'on ne pouvait expliquer qu'en termes de trahison et de corruption.

Les activistes du Mouvement macédonien de prospérité balkanique (MA.KI.V.E.), fondé en 1991, et plus tard du parti Arc-en-ciel (Ouranio Toxo / Vinozito), fondé en 1994, étaient régulièrement présentés comme "agents payés de Skopje" ou au moins comme "pro-skopjens". Ils ne pouvaient en aucun cas être des interlocuteurs reconnus, ils étaient exclus des médias et ne participaient à aucune discussion publique.

Les rares exceptions ne faisaient que confirmer la règle. Un reportage fait à Florina en octobre 1991, titré "La provocation d'une "poignée" de bilingues", affichait délibérément son parti pris ${ }^{25}$. Par ailleurs, une seule interview des activistes a été publiée en mars 1992 : le titre de l'article "Nous sommes un million de Macédoniens" 26 , tiré d'une phrase attribuée à Christos Sidiropoulos, suffisait pour discréditer d'emblée les intentions des leaders du mouvement. Cette affirmation exagérée a donné lieu à leur poursuite en justice pour "diffusion d'informations mensongères".

Malgré les pressions, le parti Ouranio Toxo a obtenu 7263 votes aux élections européennes de juin 1994 (0,10 \% au total, mais 5,7\% à Florina). Le score est faible, mais pas insignifiant. La chute du nombre de votes lors des élections suivantes démontre qu'en 1994 un nombre de slavophones a voulu protester contre l'attitude de l'État, plus que revendiquer un statut de minorité natio-

24 Ena, 11/03/92.

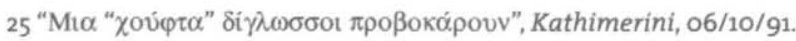

26 Ena, 11/03/92. 
nale. "Ainsi ils apprendront à nous prendre en compte", était l'explication d'un jeune électeur en Macédoine occidentale. La libéralisation de la politique de l'État après 1996 a entraîné un recul sensible du sentiment de malaise parmi la population slavophone. Le parti Ouranio Toxo a continué de se présenter aux élections, mais son retentissement a été marginal.

\section{CONCLUSION}

La crise macédonienne a été un moment de prise de conscience pour les slavophones en Grèce. Fatigués d'avoir à prouver leur loyauté nationale et de dissimuler leur spécificité au risque de sanctions, les slavophones grecs ont affiché au cours des années 1990 une volonté d'être reconnus pour ce qu'ils sont : des citoyens grecs de pleins droits, et fiers de leur culture locale.

L'amélioration spectaculaire des relations entre la Grèce et la République de Macédoine, en dépit de la question de l'appellation qui reste en suspens, a conduit à la baisse des tensions nationalistes et à la normalisation des échanges économiques et culturels entre les deux pays. En 1999, le premier ministre Ljupčo Georgevski a déclaré que les problèmes, dont celui de la minorité, seraient réglés "d'eux-mêmes" avec le développement des relations bilatérales ${ }^{27}$. Pour leur part, les députés du département de Florina s'expriment depuis longtemps en faveur du retour des derniers réfugiés de la guerre civile, qui résident toujours de l'autre côté de la frontière. Pour l'État grec c'est encore un tabou qui reste à être brisé. 